\title{
Atividade física em acadêmicos de Educação Física: um estudo longitudinal
}

\author{
Physical activity in physical education students: a longitudinal study
}

Tiago Silva dos Santos', Otávio Amaral de Andrade Leão', Jayne Santos Leite', Marcelo Cozzensa da Silva'

\section{Resumo}

O objetivo do estudo foi verificar a prática de atividades físicas e os fatores a ela associados em estudantes de Educação Física. Estudo longitudinal realizado com acadêmicos de uma Universidade Federal do sul do Brasil. Foram avaliados 81 estudantes dos cursos de licenciatura e bacharelado os quais responderam um questionário padronizado aplicado em dois períodos: início da graduação (2014) e início do segundo ano de curso (2015). O instrumento abordou questões sobre dados sociodemográficos, comportamentais e nutricional. O nível de atividade física foi avaliado nos domínios lazer e deslocamento através do International Physical Activity Questionnaire (IPAQ) versão longa. A análise estatística utilizou teste de qui-quadrado para comparação de proporções e teste de McNemar para dados pareados. A amostra apresentou maior frequência de ativos entre os indivíduos do sexo masculino, solteiros e não fumantes. Não houve diferença significativa de ativos entre cursos e entre os períodos avaliados. O período de um ano após o ingresso na universidade não mostrou diferenças na prática de atividade física, no entanto as baixas prevalências no domínio do lazer alertam para uma continuidade no acompanhamento, visto que esses indivíduos, além de se beneficiarem deste comportamento saudável, serão futuros disseminadores do mesmo.

\section{Palavras-chave}

Prevalência; Atividade física; Estudantes; Universidade; Lazer; Deslocamento.

\section{Abstract}

The aim of the study was to verify the physical activity pratice and associated factors in physical education students. A longitudinal study was conducted with academics from a federal university in southern Brazil. We evaluated 81 students of bachelor's degree and licentiate's degree who answered a standardized questionnaire applied in two periods: early graduation (2014) and beginning of the second year course (2015). The questionnaire included sociodemographic, behavioral and nutritional questions. The level of physical activity was assessed in leisure and commuting domains through the International Physical Activity Questionnaire (IPAQ) long version. The statistical analysis used chi-square test to compare proportions and McNemar test for paired data. The sample showed a bigher frequency of actives among males, singles and nonsmokers. There was no significant difference in actives between courses and between periods. The period of one year after entry into the university showed no differences in physical activity, however the low prevalence in the leisure domain warn of continuity in monitoring, since these individuals, as well as to benefit from this bealthy behavior, will be forthcoming disseminators of it.

\section{Keywords}

Prevalence; Physical activity; Students; University; Leisure-time; Transportation.

\section{Introdução}

Há muitos anos a atividade física $(\mathrm{AF})$ é reconhecidamente um fator comportamental importante para a manutenção, tratamento e melhoria da condição de saúde da população ${ }^{1-3}$. Apesar disso, estudos epidemiológicos têm demonstrado que grande parcela da população não atinge as recomendações mínimas atuais de prática de AF para benefícios à saúde $e^{1,2}$, tornando a inatividade física um dos principais pro-

1 Universidade Federal de Pelotas, Programa de Pós-Graduação em Educação Física, Pelotas, Rio Grande do Sul, Brasil. blemas enfrentados pela população mundial, com alta frequência entre os fatores de risco associado as doenças crônicas não-transmissíveis ${ }^{3}$.

Estudo realizado por Hallal et al. ${ }^{1}$ descreveu o nível de atividade física em 122 países, identificando 31,1\% de inatividade física em adultos. Quando analisados os dados de novo relatório disponibilizado sobre os níveis globais de inatividade física, evidenciou-se uma redução na prevalência da mesma para $23,8 \%^{2}$. Apesar disto, os resultados ainda são preocupantes, o que torna estudos de acompanhamentos essenciais para monitoramento destas alterações e seus fatores associados. 
No Brasil, estudo realizado por Malta et al. $(2015)^{4}$ em capitais brasileiras identificou um quadro de manutenção na prevalência de inatividade física $(16,2 \%)$ entre os anos de 2006 e 2013. Porém, ao analisar dados relativos a pratica de AF no lazer, percebe-se um aumento na prevalência, chegando a 33,8\%. Enquanto a análise do domínio de atividades de deslocamento apresentou redução, com valor de $12,1 \%$ na última análise.

Aspectos de saúde relacionados à população de jovens e adolescentes merecem avaliação especial, visto que as condutas de saúde estabelecidas durante este período podem ter impacto significante na ocorrência de doenças futuras ${ }^{5}$. Neste sentido, França e colaboradores ${ }^{6}$ descrevem que estudantes universitários geralmente apresentam uma série de comportamentos de risco para a saúde, entre os quais baixos níveis de AF. Brito et al. ${ }^{7}$ relatam que grande parte dos estudantes universitários tem adotado um estilo de vida pouco saudável, incluindo, entre esses estudantes, os acadêmicos de Educação Física (EF).

Assim como nas demais áreas de formação superior, a EF também demanda tempo de dedicação acadêmica, deslocamentos e outras implicações que podem fazer com que mesmo um estudante da área da saúde não mantenha hábitos de vida saudáveis. Alguns estudos avaliaram o nível de AF de estudantes de graduação em EF e os fatores associados ${ }^{8-10}$. Entretanto, pouco se avalia esse comportamento durante períodos da graduação. Sabendo que esse período compreende uma fase importante para manutenção de hábitos saudáveis futuros, em especial a prática de atividade física, o presente estudo teve como objetivo verificar a prática de atividades físicas e os fatores a ela associados em acadêmicos dos cursos de licenciatura e bacharelado de uma faculdade de Educação Física do sul do Brasil em diferentes períodos: no início da graduação e após um ano completo de estudo (início do segundo ano).

\section{Métodos}

Foi realizado um estudo longitudinal envolvendo todos os alunos ingressantes nos cursos de licenciatura (diurno e noturno) e de bacharelado em EF da Escola Superior de Educação Física da Universidade Federal de Pelotas no ano 2014 $(\mathrm{N}=81)$. Foram excluídos todos alunos que não estavam matriculados ou frequentando regularmente as aulas de seus respectivos cursos.

Quanto ao procedimento de coleta, primeiramente ocorreu a solicitação da lista de estudantes matriculados nos cursos de licenciatura e bacharelado com respectivos ingressos no período 2014/1 junto ao colegiado de curso da ESEF/ UFPel. Em seguida foi analisada a grade de horários das turmas com o objetivo de definir data e hora ideal para a abordagem, a qual ocorreria na primeira semana do semestre letivo. Com essa definição, foi solicitado ao professor responsável pela disciplina na qual o instrumento seria aplicado a turma, a concessão de um tempo da aula para tal finalidade.

$\mathrm{Na}$ data e horários definidos para a realização da coleta, pesquisadores colaboradores se fizeram presentes em sala de aula, esclarecendo o objetivo da pesquisa, distribuindo o termo de consentimento livre e esclarecido e, posteriormente, disponibilizando os questionários auto preenchíveis. Foi solicitado consentimento por escrito a todos os alunos para a participação na pesquisa, garantindo-se, desta forma, o direito de recusa à participação no estudo, bem como, no caso de aceite, a confidencialidade e sigilosidade das informações coletadas. Aos estudantes com idade inferior a 18 anos, o documento foi assinado pelo responsável legal do mesmo.

Os pesquisadores colaboradores mantiveram-se presente durante todo o período de preenchimento do instrumento, esclarecendo quaisquer dúvidas que pudes- 
sem vir a ocorrer antes, durante ou após a aplicação. Ao final do preenchimento individual, os questionários foram recolhidos para posterior codificação, digitação e análise dos dados.

Todos os alunos responderam a um questionário padronizado e codificado, o qual foi realizado na semana de ingresso à Universidade, mais precisamente em março de 2014, ocorrendo a reaplicação do mesmo instrumento na primeira semana do terceiro semestre letivo de seus respectivos cursos, março de 2015, caracterizando então o encerramento do ciclo de um ano de graduação.

A coleta de dados foi realizada por meio de um questionário contendo questões sociodemográficas - sexo (masculino, feminino), idade (anos completos), cor da pele (relatada pelo entrevistado), situação conjugal (casado/vive com companheiro, solteiro), filhos (sim, não), nível econômico (A/B, C, D/E) de acordo com o Critério de Classificação Econômica Brasil da Associação Brasileira de Empresas de Pesquisa; comportamentais - tabagismo (fumante e não fumante), consumo de álcool (não consome, semanalmente, eventualmente), nível de atividade física (domínios de lazer e deslocamento) e nutricional - índice de massa corporal (IMC) calculado pelo peso $(\mathrm{kg})$ referido, dividido pela altura $(\mathrm{cm})$ referida elevada ao quadrado, classificados em baixo peso/peso normal e sobrepeso/obesidade.

A principal variável de interesse, o nível de AF, foi avaliado através das seções de lazer e deslocamento, com recordatório dos últimos sete dias, do Questionário Internacional de Atividade Física (IPAQ) ${ }^{11}$, versão longa. Foram considerados ativos os alunos que atingiram, em cada domínio separadamente ou no total, 150 minutos ou mais de atividades físicas na última semana. $\mathrm{E}$ as atividades físicas vigorosas tiveram seu escore multiplicado por dois, seguindo critérios adotados por Armstrong et al. ${ }^{12}$ para explicar sua maior intensidade.

O banco de dados foi construído no programa Excel e a análise dos dados realizada através do programa STATA 13.0. O plano de análise proposto definiu as seguintes etapas: inicialmente foi realizada a análise descritiva de todas as variáveis coletadas, com cálculos de medida de tendência central e dispersão para os dados numéricos e proporções para os dados categóricos. A seguir foram realizadas as análises bivariadas, onde foi calculada a frequência de ativos conforme grupos das variáveis independentes. A diferença de proporções foi avaliada por meio do teste de Qui-quadrado e, no caso dos dados pareados, por meio do teste de McNemar. Com intuito de avaliar a significância de associações encontradas na análise bruta ajustada para as demais variáveis independentes em estudo, foi realizado um modelo ajustado utilizando a análise de regressão de Poisson. Todas variáveis entraram no modelo ao mesmo tempo sendo retiradas, uma a uma, aquelas com valor p>0,2. Para todos os testes, foi adotado um nível de significância de 5\%.

O estudo foi submetido e aprovado pelo Comitê de Ética em Pesquisa da Escola Superior de Educação Física da Universidade Federal de Pelotas recebendo aprovação sob parecer $n^{\circ}$ 1.109.109.

\section{Resultados}

A tabela 1 apresenta dados relativos ao perfil socioeconômico, demográfico, comportamental e nutricional da amostra em estudo no ingresso na Universidade e ao início do segundo ano de graduação. Pode-se verificar que o curso de licenciatura diurno foi o que apresentou maior frequência de indivíduos do sexo feminino $(64,5 \%)$, sendo esta quase o dobro da encontrada no curso de bacharelado $(31,6 \%)$. Em relação as variáveis estado civil, cor da pele e idade, pode-se perceber que, no ingresso da graduação, ambos os cursos tiveram maioria de estudantes solteiros, 
de cor da pele branca e com 20 ou menos anos de idade.

Quanto à renda, a maioria dos estudantes de cursos diurnos foi classificada nas classes C, D/E (60\% ou mais dos estudantes em ambos os períodos de coleta). Em relação ao IMC, de maneira geral, todos os cursos apresentaram aumento da categoria sobrepeso/obesidade no período investigado, sendo que nos cursos diurnos este aumento girou em torno de $10 \%$ enquanto no curso noturno foi de aproximadamente $5 \%$.

Mais de 93\% dos estudantes de todos os cursos não apresentam hábito de fumar. Porém, quando analisados os dados referentes ao consumo de álcool, verificou-se elevação próxima a 10\% na indicação de consumo semanal em ambos os cursos do turno diurno.

TABELA 1 - Perfil sociodemográfico, econômico, comportamental e nutricional da amostra em estudo no ingresso (2014) e ao início do segundo ano de graduação (2015).

\begin{tabular}{|c|c|c|c|c|c|c|}
\hline \multirow[t]{2}{*}{ Variáveis } & \multicolumn{2}{|c|}{$\begin{array}{l}\text { Licenciatura } \\
\qquad(n=31)\end{array}$} & \multicolumn{2}{|c|}{$\begin{array}{l}\text { Bacharelado } \\
\qquad(n=19)\end{array}$} & \multicolumn{2}{|c|}{$\begin{array}{l}\text { Noturno } \\
(n=31)\end{array}$} \\
\hline & $\begin{array}{l}2014 \\
\text { n (\%) }\end{array}$ & $\begin{array}{l}2015 \\
\mathrm{n}(\%)\end{array}$ & $\begin{array}{l}2014 \\
n(\%)\end{array}$ & $\begin{array}{l}2015 \\
\text { n (\%) }\end{array}$ & $\begin{array}{l}2014 \\
n(\%)\end{array}$ & $\begin{array}{l}2015 \\
n(\%)\end{array}$ \\
\hline \multicolumn{7}{|l|}{ Sexo } \\
\hline Masculino & $11(35,5)$ & $11(35,5)$ & $13(68,4)$ & $13(68,4)$ & $19(61,3)$ & $19(61,3)$ \\
\hline Feminino & $20(64,5)$ & $20(64,5)$ & $6(31,6)$ & $6(31,6)$ & $12(38,7)$ & $12(38,7)$ \\
\hline \multicolumn{7}{|l|}{ Idade } \\
\hline$<20$ anos & $19(61,3)$ & $12(38,7)$ & $10(52,6)$ & $7(36,8)$ & $10(32,3)$ & $7(22,5)$ \\
\hline 20 a 24 anos & $9(29,0)$ & $15(48,4)$ & $4(21,1)$ & $6(31,6)$ & $11(35,4)$ & $14(45,2)$ \\
\hline 25 ou + anos & $3(9,7)$ & $4(12,9)$ & $5(26,3)$ & $6(31,6)$ & $10(32,3)$ & $10(32,3)$ \\
\hline \multicolumn{7}{|l|}{ Cor da Pele } \\
\hline Branca & $28(90,3)$ & $28(90,3)$ & $14(73,7)$ & $14(73,7)$ & $27(87,1)$ & $27(87,1)$ \\
\hline Não branco & $3(9,7)$ & $3(9,7)$ & $5(26,3)$ & $5(26,3)$ & $4(12,9)$ & $4(12,9)$ \\
\hline \multicolumn{7}{|l|}{$\mathrm{IMC}\left(\mathrm{kg} / \mathrm{m}^{2}\right)$} \\
\hline $\begin{array}{l}\text { Baixo peso/ } \\
\text { peso normal }(<25)\end{array}$ & $23(74,2)$ & $20(64,5)$ & $14(73,7)$ & $12(63,2)$ & $21(67,7)$ & $20(64,5)$ \\
\hline $\begin{array}{l}\text { Sobrepeso/ } \\
\text { obesidade ( } \geq 25)\end{array}$ & $8(25,8)$ & $11(35,5)$ & $5(26,3)$ & $7(36,8)$ & $10(32,3)$ & $11(35,5)$ \\
\hline \multicolumn{7}{|l|}{ Estado Civil } \\
\hline Solteiro & $27(87,1)$ & $27(87,1)$ & $18(94,7)$ & $18(94,7)$ & $25(80,7)$ & $23(74,2)$ \\
\hline Casado/ companheiro & $4(12,9)$ & $4(12,9)$ & $1(5,3)$ & $1(5,3)$ & $6(19,3)$ & $8(25,8)$ \\
\hline \multicolumn{7}{|l|}{ Filhos } \\
\hline Sim & $2(6,5)$ & $2(6,5)$ & $1(5,3)$ & $1(5,3)$ & $3(9,7)$ & $3(9,7)$ \\
\hline Não & $29(93,5)$ & $29(93,5)$ & $18(94,7)$ & $18(94,7)$ & $28(90,3)$ & $28(90,3)$ \\
\hline \multicolumn{7}{|l|}{ Classe econômica } \\
\hline$A / B$ & $6(24,0)$ & $1(3,7)$ & $3(23,1)$ & $1(6,2)$ & $16(57,2)$ & $11(42,3)$ \\
\hline$C$ & $1(4,0)$ & $4(14,8)$ & $0(0,0)$ & $2(12,5)$ & $6(21,4)$ & $6(23,1)$ \\
\hline $\mathrm{D} / \mathrm{E}$ & $18(72,0)$ & $22(81,5)$ & $10(76,9)$ & $13(81,3)$ & $6(21,4)$ & $9(34,6)$ \\
\hline \multicolumn{7}{|l|}{ Tabagismo } \\
\hline Não fumante & $30(96,8)$ & $29(93,5)$ & $18(94,7)$ & $18(94,7)$ & $30(96,8)$ & $30(96,8)$ \\
\hline Fumante & $1(3,2)$ & $2(6,5)$ & $1(5,3)$ & $1(5,3)$ & $1(3,2)$ & $1(3,2)$ \\
\hline \multicolumn{7}{|l|}{ Consumo de álcool } \\
\hline Não consome & $9(29,0)$ & $8(25,8)$ & $5(26,3)$ & $6(31,6)$ & $5(26,3)$ & $5(26,3)$ \\
\hline Semanalmente & $2(6,5)$ & $5(16,1)$ & $2(10,5)$ & $4(21,1)$ & $1(5,3)$ & $1(5,3)$ \\
\hline Eventualmente & $20(64,5)$ & $18(58,1)$ & $12(63,2)$ & $9(47,3)$ & $13(68,4)$ & $13(68,4)$ \\
\hline
\end{tabular}


Ao analisar os dados relativos à frequência de estudantes ativos segundo as variáveis sociodemográficas, econômicas, comportamentais e nutricional (tabela 2 ), pode-se observar que, no primeiro ano, os estudantes do sexo masculino do Bacharelado foram mais ativos que as do sexo feminino $(\mathrm{p}=0,03)$. Entre os estudantes de licenciatura, a maior frequência foi encontrada entre aqueles com menos de 20 anos. Outro dado a ser destacado refere-se à classificação de ativos segundo a cor da pele dos estudantes de licenciatura, onde, no início do segundo ano de graduação (2015), nenhum indivíduo da cor não branca foi classificado como ativo.

Ainda no curso de licenciatura, pode-se perceber que indivíduos solteiros e que não possuíam filhos, apresentavam maior frequência de ativos quando comparados a seus pares. Ao verificar a associação da atividade física com o hábito de fumar no curso de licenciatura diurno, pode-se perceber que os estudantes classificados como não fumantes apresentaram maior frequência de ativos quando comparados aos fumantes em ambos os períodos.

Foi realizada análise multivariável para verificar se associações encontradas na análise bruta manteriam-se após controle para fatores de confusão. Nenhuma das associações observadas na análise bruta mantiveram-se significativas na análise ajustada (dados não apresentados em ilustrações).

TABELA 2 - Percentual de acadêmicos de graduação em Educação Física ativos nos anos de 2014 e 2015 segundo variáveis independentes em estudo $(n=81)$

\begin{tabular}{|c|c|c|c|c|c|c|}
\hline \multirow{3}{*}{ Variável } & \multicolumn{2}{|c|}{$\begin{array}{l}\text { Licenciatura diurno } \\
\qquad(n=31)\end{array}$} & \multicolumn{2}{|c|}{$\begin{array}{c}\text { Bacharelado } \\
\quad(n=19)\end{array}$} & \multicolumn{2}{|c|}{$\begin{array}{l}\text { Licenciatura noturno } \\
\qquad(n=31)\end{array}$} \\
\hline & 2014 & 2015 & 2014 & 2015 & 2014 & 2015 \\
\hline & $\%$ Ativos & $\%$ Ativos & $\%$ Ativos & $\%$ Ativos & $\%$ Ativos & $\%$ Ativos \\
\hline \multicolumn{7}{|l|}{ Sexo } \\
\hline Masculino & 100,0 & 90,9 & $92,3^{* * *}$ & 84,6 & 84,2 & 95,0 \\
\hline Feminino & 80,0 & 80,0 & 50,0 & 66,7 & 66,7 & 81,8 \\
\hline \multicolumn{7}{|l|}{ Idade } \\
\hline$<20$ anos & 94,7 & 91,7 & 70,0 & 85,7 & 70,0 & 85,7 \\
\hline 20 a 24 anos & 77,8 & 80,0 & 100,0 & 66,7 & 81,8 & 92,7 \\
\hline 25 ou + anos & 66,7 & 75,0 & 80,0 & 83,3 & 80,0 & 90,0 \\
\hline \multicolumn{7}{|l|}{ Cor da Pele } \\
\hline Branco & 85,7 & 92,9 & 85,7 & 85,7 & 74,1 & 89,3 \\
\hline Não branco & 100,0 & 0,0 & 60,0 & 60,0 & 100,0 & 100,0 \\
\hline \multicolumn{7}{|l|}{ IMC $\left(\mathrm{kg} / \mathrm{m}^{2}\right)$} \\
\hline $\begin{array}{l}\text { Baixo peso/ } \\
\text { peso normal }(<18,5 \text { a } 24,9)\end{array}$ & 82,6 & 80,0 & 78,5 & 75,0 & 81,0 & 95,0 \\
\hline $\begin{array}{l}\text { Sobrepeso/ } \\
\text { obesidade }(25 \mathrm{a}>30)\end{array}$ & 100,0 & 90,9 & 80,0 & 85,7 & 66,7 & 81,8 \\
\hline \multicolumn{7}{|l|}{ Estado Civil } \\
\hline Solteiro & $92,6^{* *}$ & 85,2 & 77,8 & 77,8 & 80,0 & 91,3 \\
\hline Casado/Companheiro & 50,0 & 75,0 & 100,0 & 100,0 & 66,7 & 87,5 \\
\hline \multicolumn{7}{|l|}{ Filhos } \\
\hline Sim & 50,0 & 50,0 & 100,0 & 100,0 & 66,7 & 100,0 \\
\hline Não & 89,7 & 86,2 & 77,8 & 77,8 & 78,6 & 89,3 \\
\hline \multicolumn{7}{|l|}{ Classe econômica } \\
\hline$A / B$ & 100,0 & 100,0 & 100,0 & 100,0 & 83,3 & 100,0 \\
\hline C & 100,0 & 100,0 & 0,0 & 100,0 & 83,3 & 66,7 \\
\hline
\end{tabular}




\begin{tabular}{|c|c|c|c|c|c|c|}
\hline $\mathrm{D} / \mathrm{E}$ & 83,3 & 77,3 & 80,0 & 76,9 & 75,0 & 90,9 \\
\hline \multicolumn{7}{|l|}{ Tabagismo } \\
\hline Não fumante & $90,0^{* * *}$ & 86,2 & 77,8 & 77,8 & 76,7 & 90,0 \\
\hline Fumante & 0,0 & 50,0 & 100,0 & 100,0 & 100,0 & 100,0 \\
\hline \multicolumn{7}{|l|}{ Consumo de álcool } \\
\hline Não consome & 77,8 & 62,5 & 100,0 & 100,0 & 60,0 & 100,0 \\
\hline Semanalmente & 100,0 & 100,0 & 100,0 & 100,0 & 0,0 & 100,0 \\
\hline Eventualmente & 90,0 & 88,9 & 66,7 & 55,6 & 76,9 & 76,9 \\
\hline
\end{tabular}

*Teste de Qui-quadrado; ** $\mathrm{p}<0,05$.

A tabela 3 apresenta a frequência de acadêmicos considerados ativos nos domínios lazer, deslocamento e total nos dois períodos de coleta. Ao comparar-se a frequência de ativos no período de lazer, deslocamento e total entre cada curso nos anos de 2014 e de 2015 pode-se observar que não houve diferença nas frequências encontradas nesses períodos. Quando comparada a frequência de ativos entre os anos de 2014 e 2015 nos diferentes domínios estudados e total de AF para o mesmo curso, também não se observou diferença estatisticamente significativa entre os períodos.

TABELA 3 - Percentual de acadêmicos de graduação em Educação Física ativos nos domínios de lazer, deslocamento e total de acordo com o curso frequentado $(n=81)$.

\begin{tabular}{lcccc}
\hline \multirow{2}{*}{ Variável } & Licenciatura $(n=31)$ & Bacharelado $(n=19)$ & Noturno $(n=31)$ & $p^{*}$ \\
\cline { 2 - 5 } & \&Ativo $\%$ & \&Ativo $\%$ & ${ }^{*}$ Ativo \% & \\
\hline Lazer 2014 & 64,5 & 52,6 & 54,8 & 0,64 \\
\hline Lazer 2015 & 54,8 & 68,4 & 61,3 & 0,63 \\
\hline $\mathrm{p}^{\#}$ & 0,36 & 0,08 & 0,59 & 0,88 \\
\hline Deslocamento 2014 & 64,5 & 57,9 & 64,5 & 0,84 \\
\hline Deslocamento 2015 & 54,8 & 63,2 & 58,1 & 0,59 \\
\hline$p^{\#}$ & 0,31 & 0,65 & 0,59 & 0,52 \\
\hline AF Total 2014 & 87,1 & 78,9 & 77,4 & 90,3 \\
\hline AF Total 2015 & 83,8 & 78,9 & 0,10 & \\
\hline$p^{\#}$ & 0,65 & 1,0 & & \\
\hline
\end{tabular}

$\mathrm{p}^{*}$ teste de Qui-quadrado para diferença entre cursos; $\mathrm{p}^{\#}$ teste de McNemar para diferença entre as coletas no mesmo curso; ${ }^{*}$ Ativo $\geq 150$ minutos de atividade física por semana.

\section{Discussão}

O presente estudo demonstrou que grande parte dos estudantes ingressantes na faculdade atingiu a recomendação de prática de $\mathrm{AF}$ para benefícios à saúde. Apesar disso, a redução na frequência observada no curso de licenciatura ao fim do primeiro ano de graduação, mesmo que não significativa, alerta para uma maior atenção a esse grupo de estudantes.

Tal comportamento pode estar ligado a fatores inerentes da rotina universitária. Silva et al. ${ }^{13}$, ao analisar o bem-estar de estudantes universitários evidenciou alguns pontos chave como possíveis motivadores à mudança de comportamento, tais como: extensa carga horária em sala de aula e exigência de estudo fora dela, uso abusivo de álcool, sono insuficiente e alimentação inadequada. Neste sentido, Joly et al. ${ }^{14}$ classifica as mudanças de comportamento em universitários em quatro categorias: 1) Mudanças acadêmicas - se referem a adaptações de ritmo de aulas e estratégias de aprendizagem, métodos de estudo e de avaliação; 2) Mudanças so- 
ciais - se referem a necessidade de aquisição de novos padrões de relacionamento, principalmente com novos indivíduos; 3) Mudanças pessoais - relativas a criação de uma identidade, personalidade, desenvolvimento integral e visão de mundo; 4) Mudanças vocacionais - onde a universidade exerce o papel de desenvolvimento da identidade vocacional, o que demanda uma maior noção de responsabilidades.

A única variável associada à prática de $\mathrm{AF}$ no curso bacharelado foi o sexo na primeira coleta, onde os estudantes do sexo masculino foram mais ativos em relação ao sexo feminino, indo ao encontro com outros estudos verificados na literatura $^{15,16}$. Estudantes solteiros do curso de licenciatura diurno apresentaram maior frequência de atividade física quando comparado aos casados/viviam com companheiro. O resultado encontrado no curso de licenciatura diurno é semelhante a de outros estudos ${ }^{9,17}$, os quais, também, identificaram que indivíduos solteiros eram mais ativos que casados/viviam com companheiro. No entanto outras investigações não encontraram associação entre AF e estado civil ${ }^{18,19}$.

Com relação ao hábito de fumar, estudantes fumantes do curso de licenciatura diurno apresentaram menor frequência de atividade física, o que contrasta com resultados de outros estudos sobre tal associação entre estudantes universitá$\operatorname{rios}^{9,20}$. Tal associação pode ser explicada, pois indivíduos que possuem comportamentos de risco, como o tabagismo, podem extrapolar este comportamento para demais esferas, como a prática de $\mathrm{AF}^{16}$.

No primeiro momento do presente estudo foi encontrada maior prevalência de AF no lazer entre estudantes do curso de licenciatura diurno (64,5\%), enquanto que, no início do segundo ano de graduação, a maior frequência foi entre estudantes do curso de bacharelado (68,4\%). Estudo realizado com estudantes de EF no primeiro ano de graduação ${ }^{10}$ apresentou maior prevalência de ativos no lazer, aproximadamente $90 \%$. Tais valores são semelhantes aos encontrados no estudo de Silva ${ }^{9}$, realizado com estudantes de EF, onde foi verificada prevalência de AF de $90,2 \%$ no curso de bacharelado e de $88,6 \%$ no curso de licenciatura. No domínio de deslocamento, observou-se redução para aproximadamente $55 \%$ na frequência de ativos em ambos os cursos de licenciatura, prevalência similar à descrita em pesquisa com estudantes portugueses do curso de $\mathrm{EF}^{21}$.

Apesar de não terem sido encontrados estudos longitudinais que verificassem a mudança de AF em estudantes universitários ao longo da graduação, alguns estudos compararam estudantes dos anos iniciais com finais do curso. Nesse sentido, Silva et al. ${ }^{22}$ não encontraram diferença na prática de $\mathrm{AF}$ entre esses períodos; em contrapartida, Fonte e Vianna ${ }^{23}$ verificaram que indivíduos com mais tempo de universidade tiveram 1,73 mais chance (IC95\%: 1,28-2,34) de apresentar baixo nível de AF em relação aos ingressantes. Bielemann et al. ${ }^{8}$ verificaram que apenas as mulheres de turmas finais do curso de EF apresentaram menor prevalência de AF no lazer comparadas as de turmas iniciais. Tal comparabilidade entre os estudos supracitados e o presente devem ser interpretadas com cautela, visto que os estudos supracitados compararam alunos e turmas diferentes nos períodos estudados enquanto o presente avaliou os mesmos alunos em dois momentos distintos.

Apesar de frequências superiores a $78 \%$ de ativos em todos os cursos no início do segundo ano de graduação, sabe-se que diferentes determinantes sociais, demográficos e ambientais, podem influenciar na variação destes resultados ${ }^{24,25}$. Tal fato evidencia a necessidade de maior investigação acerca de possíveis determinantes para adoção e manutenção da AF em acadêmicos de EF no decorrer da graduação. Outro ponto a ser observado é o curto período de acompanhamento, um ano, podendo não ser suficiente para mudanças de comportamento destes acadêmicos. 
Nesse sentido, estudo de tendência temporal com adultos nas capitais brasileiras não encontrou mudança após um ano, no entanto após quatro anos foi verificado aumento significativo na AF de lazer e diminuição também significativa na AF de deslocamento ${ }^{4}$ Sendo assim, a sequência das observações pode possibilitar uma análise e identificação dos fatores associados a mudanças de comportamento ativo nesta população.

Alguns pontos da presente pesquisa devem ser enfatizados. O processo logístico para acompanhamento dos alunos, a utilização de um questionário com instrumentos padronizados e a cautelosa coleta de dados são pontos importantes a serem destacados. As perdas e recusas da amostra podem ser consideradas como um fator limitador da pesquisa. Dos alunos regularmente matriculados e frequentando as aulas foi verificado um total de perdas/recusas de 18\% (n=27) em relação à amostra inicial. Além disso, houve um elevado número de abandonos e transferências de curso contribuindo para diminuição da amostra. Porém, o fato da utilização do real número de matrículas junto ao colegiado de curso possibilitou uma seleção coerente de amostra.

Conclui-se que a frequência total de ativos entre os estudantes de EF é elevada (acima de 78\%); entretanto é menor se levarmos em consideração o tempo de lazer (nenhuma superior a 69\%). As variáveis associadas à prática de $\mathrm{AF}$ sexo masculino, solteiro e não ser fumante. Não houve mudanças significativas na frequência de ativos entre o período de um ano da graduação, porém a redução da prática de AF apresentada em todos os domínios pelos estudantes do curso de licenciatura diurno é motivo de alerta, visto a importância da manutenção de hábitos saudáveis para uma boa qualidade de vida. Nada mais ideal que, entre estudantes de um curso de Educação Física, todos os indivíduos atinjam, pelo menos, as recomendações mínimas de AF para benefícios à saúde. Portanto, a continuidade de acompanhamento desses estudantes durante sua estada na universidade torna-se fundamental no sentido de observar e compreender os fatores que possam influenciar em possíveis mudanças de comportamento para prática de AF.

\section{Contribuição dos autores}

T.S. Santos, O.A.A. Leão e J.S. Leite supervisionaram o trabalho de campo, realizaram a tabulação dos dados, revisão de literatura, análise dos dados e redação final do artigo. M.C. Silva participou da concepção do projeto, análise de dados e todas fases de redação do artigo. Todos os autores aprovaram a versão final do manuscrito.

\section{Referências}

1. Hallal PC, Andersen LB, Bull FC, Guthold R, Haskell, W, Ekelund U. Global physical activity levels: surveillance progress, pitfalls, and prospects. Lancet 2012; 380:219-29.

2. Sallis JF, et al. for the Lancet Physical activity series working group. Progress in physical activity over the Olympic quadrennium. Lancet 2016; 388:1325-36.

3. Lee I-M, Shiroma EJ, Lobelo F, Puska P, Blair SN, Katzmarzyk PT. for the Lancet Physical activity series working group. Effect of physical inactivity on major non-communicable diseases worldwide: an analysis of burden of disease and life expectancy. Lancet 2012; 380: 219-29.

4. Malta DC, Andrade SSA, Santos MAS, Rodrigues GBA, Mielke GI. Tendências dos indicadores de atividade física em adultos: Conjunto de capitais do Brasil 2006-2013. Rev. Bras. Ativ. Fís. Saúde. 20(2): 141-51.

5. Joia LC. Perfil do estilo de vida individual entre estudantes universitários. Revista Movimento. 2010; 3:16-23. 
6. França CD, Colares V. Estudo comparativo de condutas de saúde entre universitários no início e no final do curso. Rev. Saúde Pública. 2008; 42:420-7.

7. Brito BJQ, Gordia AP, Quadros TMB. Revisão da literatura sobre o estilo de vida de estudantes universitários. Rev. Bras. de Qualidade de Vida. 2014; 06:66-76.

8. Bielemann R, Karini G, Azevedo MR, Reichert FF. Pratica de atividade física no lazer entre acadêmicos de educação física e fatores associados. Rev. Bras. Ativ. Fís. Saúde. 2007; 12:65-72.

9. Silva DAS. Nível de atividade física e fatores associados em acadêmicos de educação física de uma universidade pública do nordeste do Brasil. Rev. Bras. Ativ. Fís. Saúde. 2011; 16:193-8.

10. Mielke GI, Ramis TR, Habeyche EC, Tessmer MGS, Azevedo MR, Hallal PC. Atividade física e fatores associados em universitários do primeiro ano da universidade federal de pelotas. Rev. Bras. Ativ. Fís. Saúde. 2010; 15:57-64.

11. Craig CL, Marshall AL, Sjostrom M, Bauman, E, Booth ML, Ainsworth BE, et al. International physical activity questionnaire: 12 -country reliability and validity. Med Sci Sports Exerc. 2003; 35:1381-95.

12. Armstrong T, Bauman A, Davies J. Physical activity patterns of Australian adults - Results of the 1999 National Physical Activity Survey. Canberra: Australian Institute of Health and Welfare Canberra. Ago. 2000.

13. Silva EC. Qualidade de vida e bem-estar subjetivo de estudantes universitários [dissertação de mestrado]. São Paulo: Faculdade de Saúde da Universidade Metodista de São Paulo; 2012.

14. Joly MCRA, Santos AAA, Sisto FF. Questões do cotidiano universitário. São Paulo: Casa do Psicólogo, 2005. cap. 8, p. 159-177.

15. Colares V, Franca C, Gonzalez E. Condutas de Saúde entre universitários: diferenças entre gêneros. Cad. Saúde Pública. 2009; 25(3):521-28.

16. Palma A, Abreu RA, Cunha CA. Comportamentos de risco e vulnerabilidade entre estudantes de educação física. Rev. Bras. Epidemiol. 2007; 10(1):117-26.

17. Barros MVG, Nahas MV. Comportamentos de risco, auto-avaliação do nível de saúde e percepção de estresse entre trabalhadores da indústria. Rev. Saúde Pública 2001; 35:554-63.

18. Baretta E, Baretta M, Perez KG. Nível de atividade física e fatores associados em adultos no município de Joaçaba, Santa Catarina, Brasil. Cad. Saúde Pública. 2007. 23(7): 15951602.

19. Dias-da-Costa JS, Hallal PC, Wells JC, Daltoe T, Fuchs SC, Menezes AM, et al. Epidemiology of leisure-time physical activity: a population-based study in southern Brazil. Cad. Saúde Pública. 2005; 21:275-82.

20. Rodrigues ESR, Cheik NC, Mayer AF. Nível de atividade física e tabagismo em universitários. Rev. Saúde Pública 2008;42(4):672-8.

21. Silva PAC, Vaz VPS, Silva MC. Nível de atividade física no lazer e deslocamento e fatores associados em alunos de educação física em Coimbra-Portugal. Rev. Bras. Ativ. Fís. Saúde. 2015; 20(6):559-60.

22. Silva GSF, Bergamaschine R, Rosa M, Melo C, Miranda R, Bara Filho M. Avaliação do nível de atividade física de estudantes de graduação das áreas saúde/biológica. Rev. Bras. Med. Esporte. 2007; 13(1):39-42.

23. Fontes ACD, Vianna RPT. Prevalência e fatores associados ao baixo nível de atividade física entre estudantes universitários de uma universidade pública da região NordesteBrasil. Rev. Bras. Epidemiol. 2009; 12(1):20-9.

24. Deliens T, Deforche B, De Bourdeaudhuij I, Clarys P. Determinants of physical activity and sedentary behaviour in university students: a qualitative study using focus group discussions. BMC Public Health. 2015; 15:201.

25. Craig CL, Russel SJ, Cameron C, Bauman A. Twenty-year trends in physical activity among canadians adults. Can J Public Health. 2004; 95:59-63.

Endereço para

Correspondência

Tiago Silva dos Santos

tiago_ss@outlook.com.br
Escola Superior de Educação Física,

Universidade Federal de Pelotas

Rua Luiz de Camões 625

CEP 96055-630, Pelotas, RS, Brasil

Fone: 53 3273-2752
Recebido

Revisado

05/04/2016

$21 / 08 / 2016$

$11 / 11 / 2016$

Aprovado 24/11/2016 